\title{
Abflusskonzentration - wie Abfluss in der Fläche gebündelt und beschleunigt wird
}

5.1 Grundprinzipien und wesentliche Einflussgrößen - 96

5.2 Abflusstypen und Fließpfade in kleinen, ländlichen Einzugsgebieten - 97

5.3 Methoden zur Abschätzung von Fließgeschwindigkeit und Fließzeit - 102

Literatur - 110 
Dieses Kapitel befasst sich mit der zunehmenden Bündelung und der damit einhergehenden Beschleunigung des Abflusses. Zunächst werden allgemeine Grundprinzipien und Einflussgrößen vorgestellt ( $\triangleright$ Abschn. 5.1). Dann folgt eine kurze Beschreibung typischer Abflusstypen und Fließpfade in kleinen, ländlichen Einzugsgebieten ( Abschn. 5.2). Darauf aufbauend werden hydraulische Grundlagen und Methoden zur Bestimmung von Fließgeschwindigkeit und Fließzeit beschrieben ( $\triangleright$ Abschn. 5.3). Insbesondere die Konzentrationszeit ist wichtig. Sie ist die Zeit, die Oberflächenabfluss benötigt, um von der Wasserscheide bis zu einem definierten Bezugspunkt, z. B. einer Ortschaft, zu fließen. Der Bezugspunkt richtet sich nach den örtlichen Gegebenheiten und wird je nach Fragestellung festgelegt. Die Konzentrationszeit lässt sich über die sogenannte Geschwindigkeitsmethode aus der Summe der Fließzeiten des Abflusses in verschiedenen Abflussabschnitten abschätzen. Die vorgestellten Ansätze bilden die Grundlage zur Ableitung von Maßnahmen zur Abflussverzögerung bzw. zur Abschätzung ihrer Wirkung ( Kap.6). - Anhang 8.2 ist eine Ergänzung dieses Kapitels und fasst hydraulische Eigenschaften ausgewählter Fließpfade zusammen. Zur Veranschaulichung der Methoden enthält $>$ Kap. 7 ein Anwendungsbeispiel, bei dem die Einflüsse von Wegseitengräben und der Bewirtschaftung auf Fließzeit und Scheitelabfluss in einem kleinen Einzugsgebiet abgeschätzt wird.

\subsection{Grundprinzipien und wesentliche Einflussgrößen}

Abfließender Regen wird entlang seiner Fließstrecke an der Wasserscheide beginnend und bis in die Gewässer zunehmend gebündelt. Die Länge und Dichte der Fließpfade sowie deren hydraulische Eigenschaften bestimmen die Geschwindigkeit, mit der Wasser aus der Fläche abgeleitet wird, und damit die Fließ- bzw. Konzentrationszeit und das Niveau des Scheitels einer Abflussoder Hochwasserwelle.

Die Fließpfade und ihre hydraulischen Eigenschaften sind vielfältig. Einflussgrößen von übergeordneter Bedeutung sind das Gefälle, die Rauheit des Untergrunds und die Querschnittsgeometrie des Fließpfades, die den hydraulischen Radius bestimmt. Das Gefälle ergibt sich primär aus der Topographie der Oberfläche. Die Rauheit hängt stark von der Landnutzung ab, wobei versiegelte Flächen besonders glatt und dicht bewachsende Flächen mit unregelmäßigem Untergrund (z. B. Auwälder) hydraulisch sehr rau sind. Glatte Flächen beschleunigen, raue bremsen den Abfluss. Für den Einfluss der Querschnittsgeometrie gilt, dass die Reibung hoch und damit der Abfluss langsam ist, wenn die Fließtiefe im Vergleich zur Fließbreite sehr gering ist. Durch die zunehmende Bündelung des Abflusses von der Wasserscheide bis in die Gewässer nehmen die Fließtiefe und damit die Fließgeschwindigkeit daher im Allgemeinen $\mathrm{zu}$, obwohl das Gefälle geringer wird. Weitere Einflüsse auf die Abflusskonzentration in der Fläche ergeben sich aus Strukturen, die Wasserflüsse lenken oder eigene Fließwege erzeugen. Dazu können beispielsweise Gräben, Feldraine und Schlaggrenzen, Wege, Verrohrungen oder Drainagen, aber auch Hecken und Mulden gehören [1]. Steht die Abflusskonzentration auf einzelnen, landwirtschaftlichen Schlägen im Fokus, sind Richtung und Art der Bewirtschaftung wichtig für die Fließrichtung.

Entsprechend vielseitig sind auch die Ansatzpunkte zum Bremsen des Abflusses: Sie erstrecken sich über den kompletten Fließpfad von der Wasserscheide bis in die Gewässer, und sie umfassen ein breites Repertoire an Maßnahmen, wie Saatund Bodenbearbeitungstechniken, die Erschließung und Gliederung der Flur, den Wegebau, die Gestaltung von Gräben zur Wasserführung oder den Einsatz von Rohren und Drainagen. Dadurch wirken auch zahlreiche Akteure mit. 


\subsection{Abflusstypen und Fließpfade in kleinen, ländlichen Einzugsgebieten}

Übersteigt die Niederschlagsintensität die Infiltrationsrate (s. Kap.4), entsteht in der Fläche Schichtabfluss. Dies ist ein meist wenige Millimeter starker, flächenhaft auftretender und nicht zusammenfließender Abfluss auf der Bodenoberfläche. Er ist insbesondere auf landwirtschaftlichen Flächen auch für Erosion und Stofftransport bedeutsam. Der Schichtabfluss dominiert an der Wasserscheide oder unterhalb einer wasserableitenden Struktur, bündelt sich dann aber üblicherweise rasch, d.h. häufig nach $50 \mathrm{~m}$ spätestens meist nach $100 \mathrm{~m}$, in fein-verästelten Rillen und Rinnen. Die Fließstrecke, nach der dies erfolgt, hängt stark von der Stabilität und Beschaffenheit der Oberflächenstruktur ab. Durch die Art der angebauten Früchte und die Bewirtschaftungsweise variiert dies jahreszeitlich. Dennoch bilden sich auf Ackerschlägen oft typische Abflussmuster aus. Diese Rillen und Rinnen entwässern dann über geomorphologisch bedingte Hangmulden weiter. In Hangmulden können an Schlaggrenzen und Hangfüßen bei Starkregen bereits Abflussraten von mehreren hundert Litern pro Sekunde auftreten. Die Fließpfade in der Fläche lassen sich daher in drei Abschnitte untergliedern (• Abb. 5.1):

1. das flächenhafte Fließen (Schichtabfluss),

2. der Abfluss in verästelten Rinnen und Rillen (flacher konzentrierter Abfluss) und

3. der Hangmuldenabfluss in Tiefenlinien (flacher, stärker konzentrierter Abfluss).

Grundsätzlich gilt: Je rauer die Oberfläche, desto langsamer die Fließgeschwindigkeit und desto schwächer auch die Abflusskonzentration, weil das Einschneiden der Rillen und Rinnen später erfolgt. In Wäldern und auf Grünland ist die Bedeckung durch Streu und auf dem Boden aufliegende

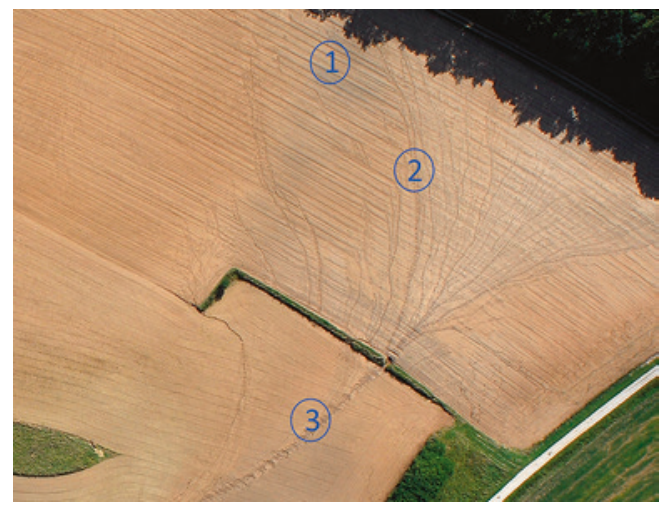

- Abb. 5.1 Abfolge und zunehmende Konzentration des Oberflächenabflusses auf einem querbearbeiteten Ackerschlag vom Schichtabfluss (1), zum Fließen in verästelten Rillen und Rinnen (2) und dem Abfluss in Hangmulden (3) nach einem Starkregenereignis von $54 \mathrm{~mm} \mathrm{~h}^{-1}$ am 21.08.2012. (Quelle: W. Bauer, Agroluftbild)

Pflanzenteile meist hoch und ändert sich im Jahresverlauf kaum. Dadurch ist die mittlere Abflussgeschwindigkeit des Oberflächenabflusses gering und die Strecke ist lang, die Oberflächenabfluss als flacher (und langsamer) Schichtabfluss zurücklegt. Da der Abfluss sich nicht einschneiden kann, entfällt das Fließen in Rinnen und Rillen bei Wald und Grünland oft ganz. Dadurch wird die Strecke des Schichtabflusses besonders bei Grünland viel länger als auf Ackerflächen, da der Schichtabfluss hier direkt in den Hangmuldenabfluss übergeht. Entsprechend vermindern Wald und Grünland Abflussbildung, Abflussgeschwindigkeit und Abflusskonzentration.

Besonders problematisch für die Entstehung von Oberflächenabfluss und Erosion sind dagegen einheitlich mit Reihenkulturen (z. B. Kartoffel, Soja, Mais, Zuckerrübe oder Sonnenblumen) bestellte, langgezogene Hänge. Bei Starkregenereignissen, vor allem im Mai und Juni, verschlämmen diese durch den späten Reihenschluss oft rasch und bieten dadurch keinen wirksamen Schutz vor einer effektiven Abflusskonzentration. Es bilden 

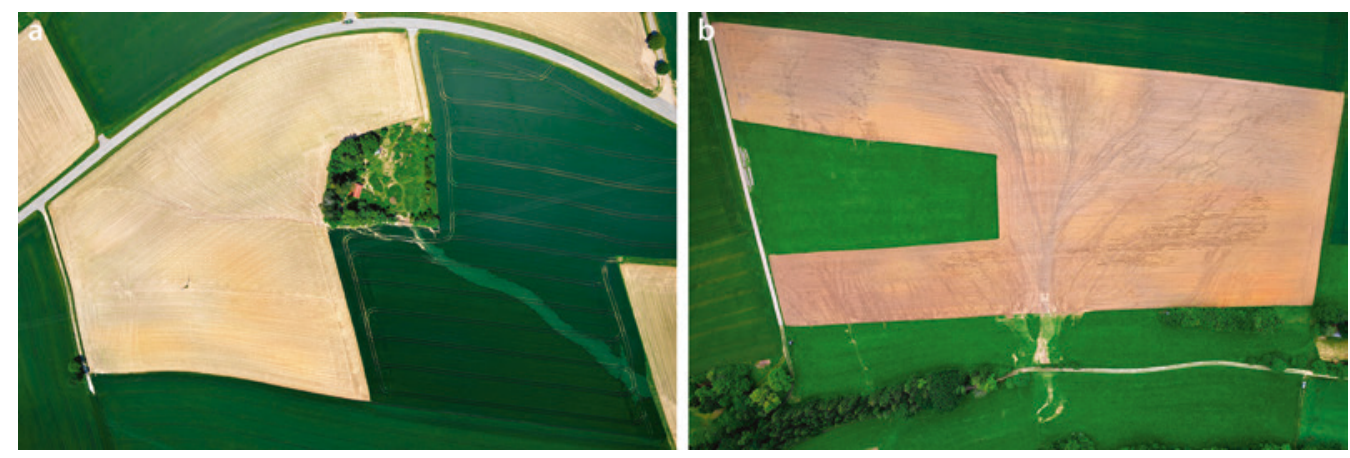

- Abb. 5.2 Massive Bodenabschwemmungen aus Maisfeldern und Abfluss in Tiefenlinien nach Starkregenereignissen vom 06.06.2016 (a) und 04.06.2016 (b) im Gebiet zwischen Hemau und Laaber (Bayern). In der linken Hälfte des rechten Luftbildes wurden Abflusskonzentration (kein baumartig verzweigtes Netz aus Fließpfaden) und Erosion weitgehend durch die Grünlandparzelle verhindert. (Quelle: W. Bauer, Agroluftbild Obertraubling in [3])

sich deutliche Fließmuster, wie sie Luftbildaufnahmen von Maisfeldern (• Abb. 5.2), die etwa 10-jährlichen Starkregenereignissen ausgesetzt waren, anschaulich zeigen [2].

Sind lineare Landschaftselemente vorhanden, die den Abfluss ableiten, bevor er sich in Hangmulden sammelt, können die Fließwege zwischen einzelnen Feldern und dem Gewässernetz vielfältig sein. Üblicherweise erfolgt der Abfluss entweder über Schlaggrenzen hinweg der Topographie folgend in Hangmulden durch angrenzende Felder oder er wird durch Ranken, Hecken und Wege oder in Wegseitengräben und Verrohrungen umgeleitet und auf konstruierten Entwässerungspfaden zum nächsten Vorfluter geleitet (• Abb. 5.3). Die Bedeutung dieser linearen, den Oberflächenabfluss leitenden Strukturen ist den handelnden Akteuren oft nicht bekannt und ihre Effekte werden in den meisten Studien nicht berücksichtigt [1]. Detaillierte Analysen kleiner Einzugsgebiete, z. B. aus dem Schwimmbachgebiet [4] oder aus Österreich [5], belegen jedoch, dass oft $40 \%$ der Fläche kleiner Einzugsgebiete nicht über topographisch bedingte Fließpfade entwässern, sondern über konstruierte Fließwege. Diese bestätigen auch eigene Erhebungen im Tertiärhügelland, wo fast $60 \%$ der Entwässerungswege in künstlichen Gräben und
Rohren verlaufen. Solche Strukturen könnten prinzipiell, verglichen mit natürlichen Fließpfaden, den Abflussweg verlängern. Heute sind künstlich angelegte Strukturen allerdings meist kürzer und auch glatter, wodurch konstruierte Fließpfade in der Regel den Abfluss beschleunigen [6]. Ein weiterer negativer Effekt der beschleunigten Ableitung von Oberflächenwasser besteht darin, dass das Puffer- und Retentionspotenzial in der Fläche und im Boden nicht oder nur in reduzierter Form genutzt wird, wodurch lineare Entwässerungsstrukturen auch erheblich zur stofflichen Gewässerbelastung beitragen [5].

Das Netz von Fließpfaden und Entwässerungswegen in kleinen Einzugsgebieten wird also nicht nur von der Topographie, sondern auch stark von der Gliederung der Flur und ihrer Erschließung durch Wegenetz und Gräben, aber auch von Drainagen und Verrohrungen geprägt. Lineare Strukturen lenken die auftretenden Wasserflüsse erheblich und beeinflussen damit, wie viel Oberflächenabfluss infiltrieren und zurückgehalten werden kann und wie schnell der Abfluss in die Gewässer gelangt. Dadurch können lineare Strukturelemente einen wichtigen Beitrag zum Erosionsschutz und zur Sicherung der stofflichen Wasserqualität leisten oder das Gegenteil bewirken. Die Förderung des Wasserrückhalts in der Fläche und die 

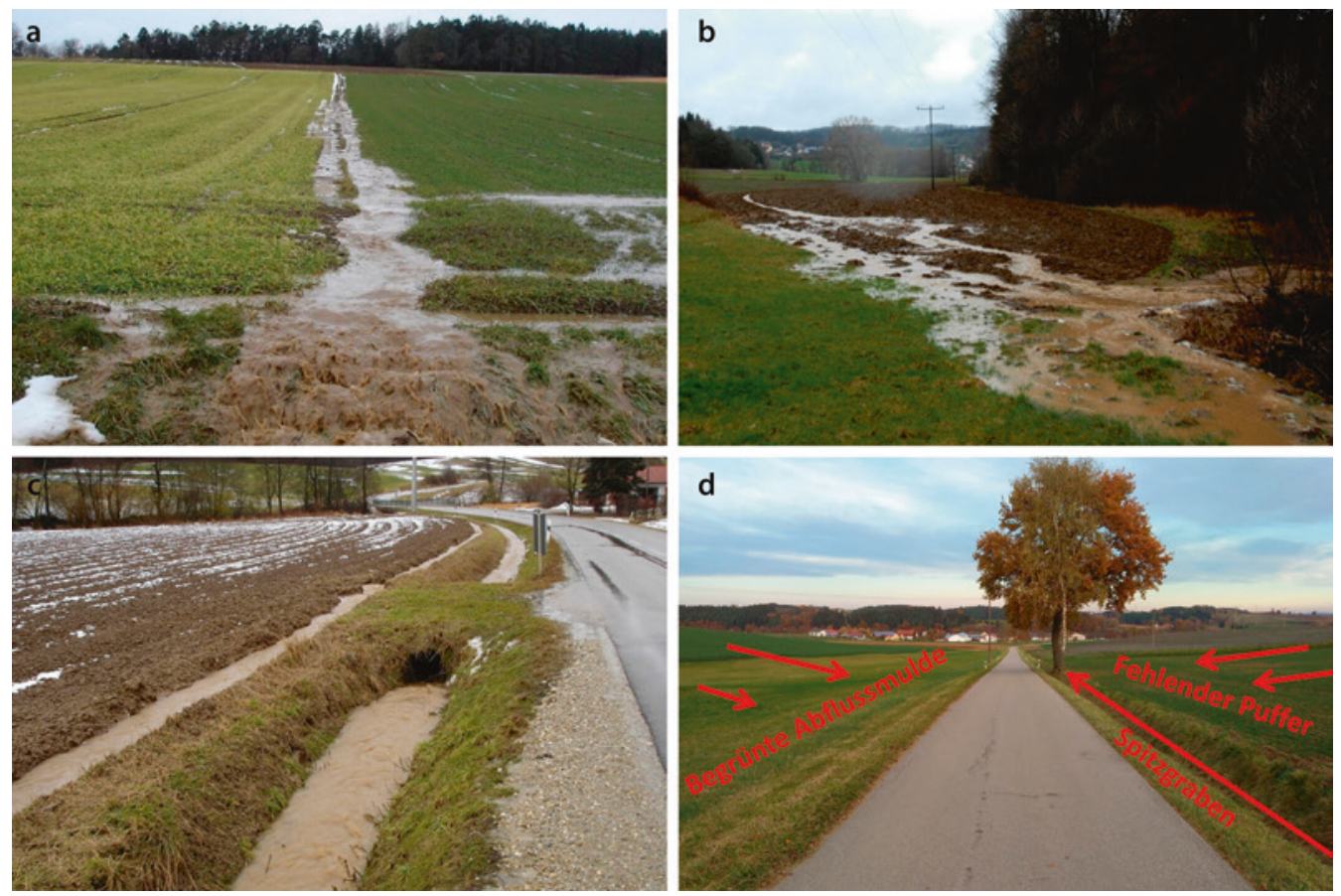

- Abb. 5.3 Fließpfade des Abflusses zwischen Feld und Gewässernetz können vielfältig sein und beispielsweise an Schlaggrenzen (a), in Tiefenlinien (b) oder entlang von Bewirtschaftungsstrukturen wie Ackerrandfurchen oder in Wegseitengräben verlaufen (c). Insbesondere Wegseitengräben können topographisch bedingte Entwässerungswege unterbrechen und durch effiziente Fließwege ersetzen (d). (Bildquellen: boden:ständig (a und c), S. Seibert (b und d))

Minimierung der stofflichen Gewässerbelastung setzen daher neben einem Verständnis für die Prozesse der Abflussbildung auch Kenntnisse über die Fließwege zwischen Wasserscheide und Gewässer voraus. Da viele Strukturen, insbesondere die Verläufe von Gräben, kleinen Wällen oder Verrohrungen selbst aus hochauflösenden Geländemodellen kaum zuverlässig extrahiert werden können, sind Gebietsbegehungen häufig unerlässlich, um den Verlauf der Fließwege zu erfassen.

Unter den linearen Strukturen sind Wege und Wegseitengräben von besonderer Bedeutung. Sie beeinflussen die Abflusskonzentration kleiner Einzugsgebiete stark und nachhaltig, indem sie den Anteil der versiegelten Fläche erhöhen, die Landschaft zerschneiden und dadurch natürliche Entwässerungswege und Einzugsgebietsgrenzen verändern sowie zusätzliche Fließpfade schaffen [7]. Aus der amtlichen Statistik ist bekannt, dass beispielsweise in Bayern rund $210.000 \mathrm{~km}$ Feld- und rund $100.000 \mathrm{~km}$ Waldwege vorhanden sind, wovon rund $60 \%$ erst seit den 1960er-Jahren gebaut wurden [8]. Dies entspricht etwa der dreifachen Länge des in Bayern vorhandenen Fließgewässernetzes. Damit wird schon allein aufgrund der Länge des Wegenetzes und der dadurch versiegelten oder stark verdichteten Fläche der Wasserhaushalt verändert. Gleichzeitig gehen mit einer Veränderung des Wegenetzes fast immer Veränderungen der Landnutzung und -struktur einher. Beides kann kaum unabhängig untersucht werden. Die wenigen vorhandenen quantitativen Einschätzungen beruhen auf Modellierungsstudien, Messkampagnen aus sehr kleinen Einzugsgebieten und dem Vergleich flurbereinigter und nicht flurbereinigter Einzugsgebiete. Für Letztere ist aus Südwestdeutschland 
bekannt, dass in flurbereinigten Gebieten der Gesamtabfluss im Jahr etwa 30\% höher und die Scheitelabflüsse bis zu 4-fach größer sein können als in nicht flurbereinigten Gebieten [9, 10]. Dies deckt sich mit Untersuchungen aus der Schweiz, wo der Einfluss der Flurbereinigung, vor allem der Bau von Wegen, Wegseitengräben und Dränen, auf das Abflussverhalten von drei kleinen (130, 180 und 380 ha) landwirtschaftlichen Einzugsgebieten untersucht wurde [11]. In Modellierungsstudien konnte für das Wahnbacheinzugsgebiet gezeigt werden, dass der Abfluss bei Berücksichtigung des Wegenetzes um $20 \%$ ansteigt [12]. Für kleine, terrassierte Weinbaugebiete im Kaiserstuhl wird beschrieben, dass der Hauptanteil des Oberflächenabflusses durch befestigte Wege entsteht und dass der Anteil des Oberflächenabflusses proportional zur Vergrößerung der versiegelten Flächen zunimmt [13].

Eine wesentliche Eigenschaft von Wegen besteht also darin, dass sie, durch die Bereitstellung versiegelter Flächen, immer und unabhängig von der Niederschlagshöhe zum Abflussgeschehen beitragen [7]. Bei kleinen Niederschlagsereignissen ist ihr Beitrag überproportional groß, weil da die übrige Fläche noch keinen Abfluss liefert. Bei großen Ereignissen beruht dagegen die Hauptwirkung von Straßen und Wegen darauf, dass sie zusammen mit den Seitengräben die Abflussgeschwindigkeit erhöhen, und der Scheitelabfluss steigt. Da Straßen und Wege fast immer mit Wegseitengräben ausgeführt werden, teilweise auch beidseitig (s. Kap. 7), hat der Wegebau ein sekundäres Gewässernetz geschaffen. Wenn Feld- und Waldwege dreifach länger sind als das primäre Gewässernetz, bedeutet dies, dass das sekundäre Gewässernetz drei- bis sechsfach länger sein muss als das primäre. Dieses sekundäre Gewässernetz ist die überwiegende Zeit des Jahres inaktiv und wird daher häufig nicht als solches wahrgenommen. Auch ist dieses sekundäre Gewässernetz in der Regel nicht systematisch erfasst und nicht in Karten dargestellt. Daher wird seine Bedeutung stark unterschätzt [14].

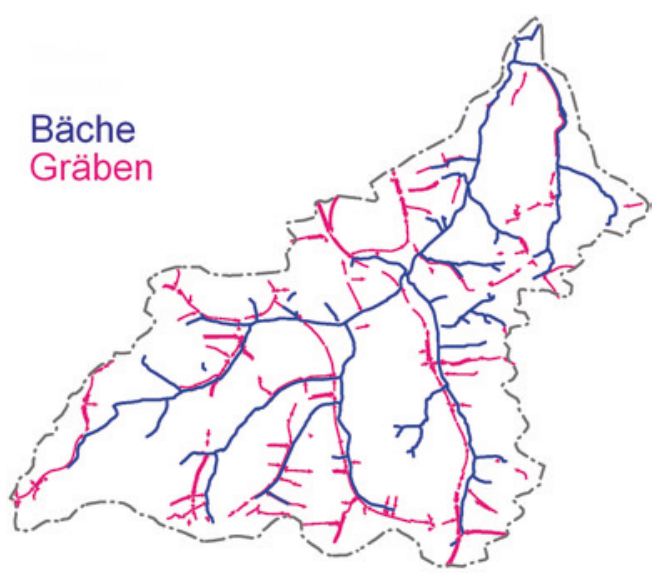

- Abb. 5.4 Netzwerk aus Gräben (74 km) zusätzlich zum Netz der Bäche (54 km) im 47 km² $^{2}$ großen Einzugsgebiet des Schwimmbachs bei Marklkofen an der Vils [15]

Detaillierte Erfassungen von Wegseitengräben in Beispielgebieten verdeutlichen jedoch, dass Gräben die Landschaft vielerorts sehr feingliedrig erschließen und parallel $\mathrm{zu}$ den Gewässern III. Ordnung ein sekundäres Entwässerungsnetz entstand (• Abb.5.4; s. a. - Kap.7). Beim Auftreten von Oberflächenabfluss wird es aktiviert und ist dann entsprechend seiner Länge bedeutsamer als das Netz dauerhaft wasserführender Gewässer. Für den erosionsbedingen Sedimenttransport ist außerdem bedeutsam, dass Abfluss, der von einem Wegseitengraben abgefangen und dem Gewässer zugeleitet wird, keinen Gewässerrandstreifen durchfließt. Damit stellen Wegseitengräben einen sehr effektiven Bypass dar, der die meisten Gewässerrandstreifen außer Funktion setzt.

Besonders anschaulich lassen sich die Effekte auf Abflussbildung und -konzentration bei Wegen, die schräg zum Hang verlaufen, illustrieren (• Abb. 5.5 oben links). In diesem Fall können Wege eine Vielzahl natürlicher, in Gefällerichtung verlaufender Fließpfade unterbrechen und das Wasser großer Einzugsgebietsflächen sammeln und konzentriert zum Tiefpunkt ableiten. Dies ist insbesondere 


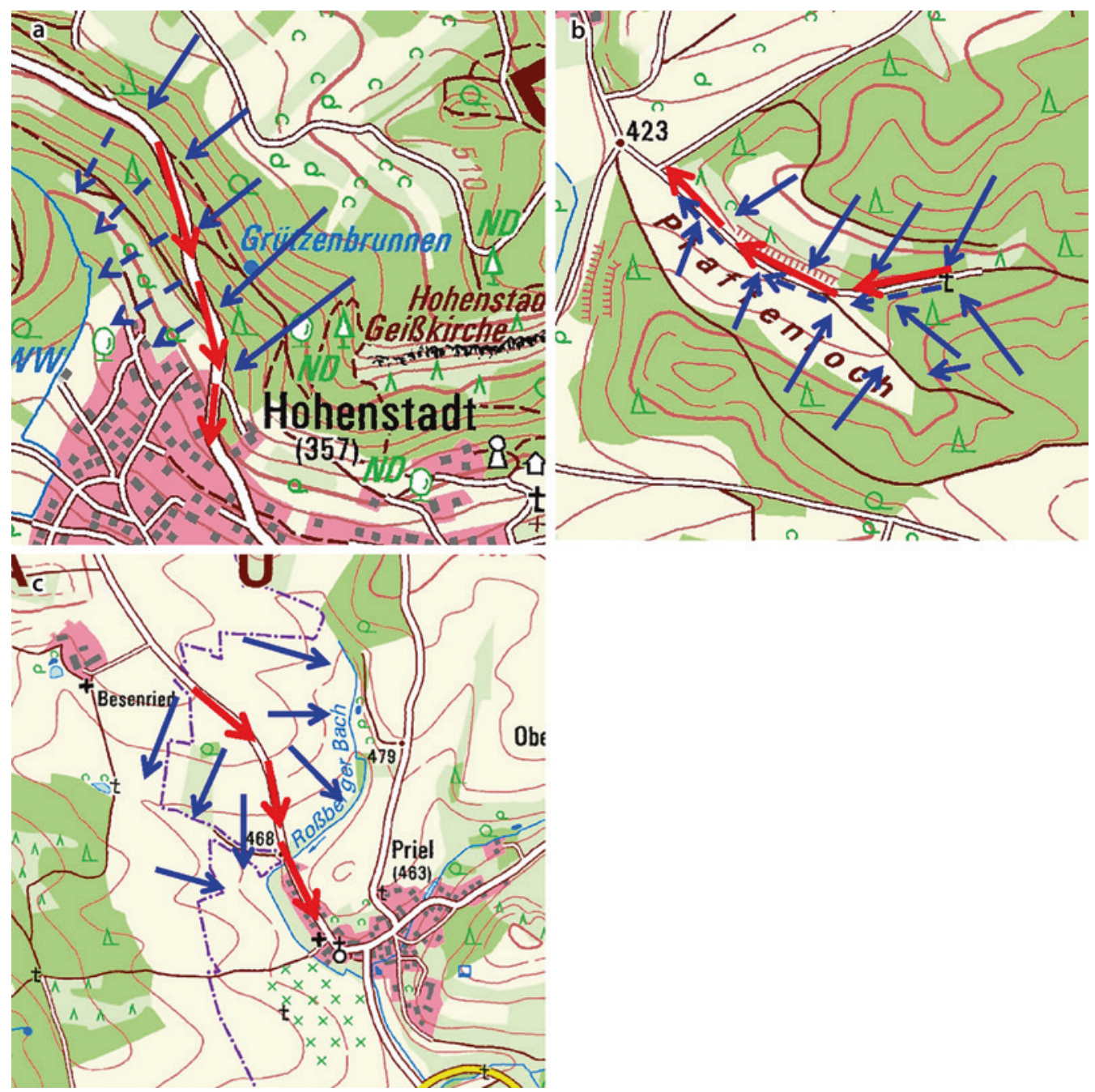

- Abb. 5.5 Verlauf natürlicher (blaue Pfeile), unterbrochener (blau gestrichelte Pfeile) und durch den Wegebau künstliche geschaffener Entwässerungswege (rote Pfeile). Dargestellt sind schräg zum Gefälle verlaufende Wege (a), die den Abfluss großer Einzugsgebiete sammeln und zu einem einzigen Tiefpunkt hin ableiten können. Wege in der Tiefenlinie (b) sind sehr weit verbreitet und können den Abfluss auf der Straße oder in wegbegleitenden Gräben stark beschleunigen. Wege auf der Wasserscheide bzw. mit einem Verlauf auf dem Hangrücken (c) beeinflussen die natürliche Entwässerung kaum. Lediglich auf dem Weg selbst kommt es durch das Gefälle zu einer starken Beschleunigung des mengenmäßig aber geringen Abflusses (Geobasisdaten: Bayerische Vermessungsverwaltung)

bei Waldwegen problematisch, da im Wald ein großer Teil des Abflusses bei Starkregen oder Schneeschmelze als Zwischenabfluss langsam im Boden fließt. Durch den Einschnitt der schräg verlaufenden Wege tritt dieser Zwischenabfluss aus und wird zu Oberflächenabfluss, der im Wegseitengraben um ein Vielfaches schneller abgeleitet wird [16] (vgl. auch $>$ Anhang 8.7).

Problematisch können auch Wege in der Tiefenlinie sein, wenn der Weg selbst oder ein hydraulisch effektiv gestalteter Seitengraben das Wasser sammelt und ableitet (- Abb. 5.5 oben rechts). Unter solchen 
Bedingungen wird der Abfluss fast immer (deutlich) beschleunigt, da der konstruierte Entwässerungsweg in nahezu allen Fällen hydraulisch effektiver gestaltet ist als ein Fließpfad, der der natürlichen Topographie folgend durch einen bewachsenen Taleinschnitt führen würde.

Günstig sind dagegen Wege entlang der Wasserscheide und auf Hangrücken (- Abb. 5.5 unten links). Diese verfügen zwar im unteren Bereich über ein hohes Gefälle, was $\mathrm{zu}$ einer Beschleunigung des Abflusses führt, dafür ist ihr Einzugsgebiet aber sehr klein, wodurch ihre Bedeutung für die Gebietsentwässerung gering bleibt. Bemerkenswert ist, dass früher vermutlich viele Wege so geführt wurden (s. A Anhang 8.7), wohl auch, um die damals unbefestigten Wege vor Erosion durch Wasser zu schützen. Durch das kleine Einzugsgebiet bei dieser Wegführung war kein Wegseitengraben erforderlich. Vor allem in den 1970er-Jahren wurden dann die Wege in die vorher begrünte Tiefenlinie gelegt, und damit musste der Wegseitengraben als integraler Bestandteil des Wegebaus eingeführt werden. Die abflussbremsendste Art, Wasser abzuleiten, nämlich entlang einer flachen Grünlandmulde, wurde ersetzt durch die schnellste und damit auch hochwasserförderndste Art, nämlich einen effizienten Graben.

\subsection{Methoden zur Abschätzung von Fließgeschwindigkeit und Fließzeit}

Dem Charakter eines Handbuchs über Planungsmethoden folgend, werden hier einfache, etablierte Berechnungsansätze wie die Gauckler-Manning-Strickler-Gleichung (GMS-Gleichung) vorgestellt. Dabei müssen teils stark vereinfachende Annahmen getroffen werden. Dazu zählt, dass der Abfluss als konstant und gleichförmig betrachtet wird (stationäre Bedingungen). Dies ist aber grundsätzlich nicht der Fall, da jede Welle sich zumindest aufbaut und dann wieder abflacht. Um diese instationären Bedingungen korrekt abzubilden, wären Flachwassergleichungen (Saint Venant) erforderlich, wie sie üblicherweise in hydrodynamischen Modellen implementiert sind. Aufgrund ihrer Komplexität erfordern diese Modelle jedoch entsprechendes Wissen, Software und hinreichend hochaufgelöste und genaue Daten. Trotz dieser Einschränkung können mit stationären Betrachtungen für viele praktische Fragestellungen hinreichend genaue Lösungen ermittelt werden. Das gilt zum Beispiel für die Bestimmung der Konzentrationszeit bei kurzen schauerartigen Starkregen. Da die Annahme konstanter Abflussverhältnisse das Aufbauen der Welle zu Beginn eines Ereignisses vernachlässigt, wird die tatsächliche Konzentrationszeit unterschätzt. Aus gleichem Grund werden die Unterschiede zwischen Flächen unterschätzt, die sich in ihrer Fließgeschwindigkeit unterscheiden. Da das Aufbauen einer Welle bei langsamer Fließgeschwindigkeit deutlich länger dauert als bei hoher Fließgeschwindigkeit, ist auch die Unterschätzung größer. Die auf der GMSGleichung beruhenden Berechnungen stellen daher eine konservative Annahme hinsichtlich der Unterschiede zwischen verschiedenen Varianten dar. Der Vorteil der GMS-Gleichung ist, dass schnell und mit relativ geringem Aufwand die Abflussgeschwindigkeit abgeschätzt und der Einfluss eines Fließpfades auf den Abfluss beschrieben werden kann. Auch kann so ermittelt werden, wie Fließpfade gestaltet werden müssen, um möglichst abflussbremsend $\mathrm{zu}$ wirken. Für die Anwendung der GMS-Gleichung müssen die Fließpfade in einigermaßen einheitliche Abschnitte unterteilt werden. Dies sind meist - von der Wasserscheide ausgehend - der Schichtabfluss, der flache konzentrierte Abfluss, der Abfluss in Hangmulden und schließlich der Abfluss in Gerinnen und Gewässern. Für diese Abschnitte werden dann die hydraulischen Kenngrößen ermittelt (s. Abschn. 5.2), wodurch sich Fließzeit und -geschwindigkeit in den einzelnen Abschnitten und aus deren Summe die Konzentrationszeit abschätzen 
lässt. Das Verfahren wird als Geschwindigkeitsmethode bezeichnet. In $>$ Kap. 7 befindet sich ein Rechenbeispiel.

\subsubsection{Fließwege im Einzugsgebiet}

Ausgangspunkt zur Beschreibung der Abflusskonzentration und zur Planung von Maßnahmen ist die Erfassung der dominanten Fließprozesse im Einzugsgebiet. Dies erfordert eine Erfassung der wichtigsten oberirdischen Fließwege von der Wasserscheide bis ins Gewässernetz. Dazu bieten sich eine Feldkartierung oder die Auswertung digitaler Geländemodelle an (idealerweise wird beides kombiniert). Fließpfadalgorithmen sind heute in allen gängigen Geographischen Informationssystemen (GIS) implementiert und erlauben es, Fließpfadmuster in der Fläche schnell und effizient aus Geländemodellen abzuleiten (• Abb.5.6). Voraussetzung dafür sind hochauflösende (Rasterweite $\leq 5 \mathrm{~m}$ ) und von Artefakten bereinigte Geländemodelle.
Artefakte sind häufig problematisch und Strukturen wie Wegseitengräben, verrohrte Abschnitte, kleine Brücken und Wälle, Drainagen oder andere den Abfluss lenkende Strukturen, wie Bordsteinkanten oder Feldraine, werden nicht oder nicht korrekt dargestellt. Auch können sich Fließwege im Jahresverlauf oder in Abhängigkeit von Bodenbewirtschaftung und Regenintensität ändern [17], was ggf. berücksichtigt werden muss, wenn konkrete Ereignisse rekonstruiert werden sollen. Eine Gebietsbegehung zur Verifizierung der Ergebnisse digitaler Ableitungen ist daher im Allgemeinen unerlässlich. Eine Begehung bietet darüber hinaus die Möglichkeit, Spuren von Stofftransport wie Erosionsrinnen, verfüllte oder erodierte Gräben, überspülte Wege oder Feldraine und dergleichen $\mathrm{zu}$ erfassen. Dies liefert oft wertvolle Informationen zum Verständnis der lokalen Abflussverhältnisse und Hinweise für Maßnahmen zur Abflussminderung. Praktische Empfehlungen zur Kartierung von Fließwegen sind in $>$ Anhang 8.8 zusammengestellt. Sind quantitative

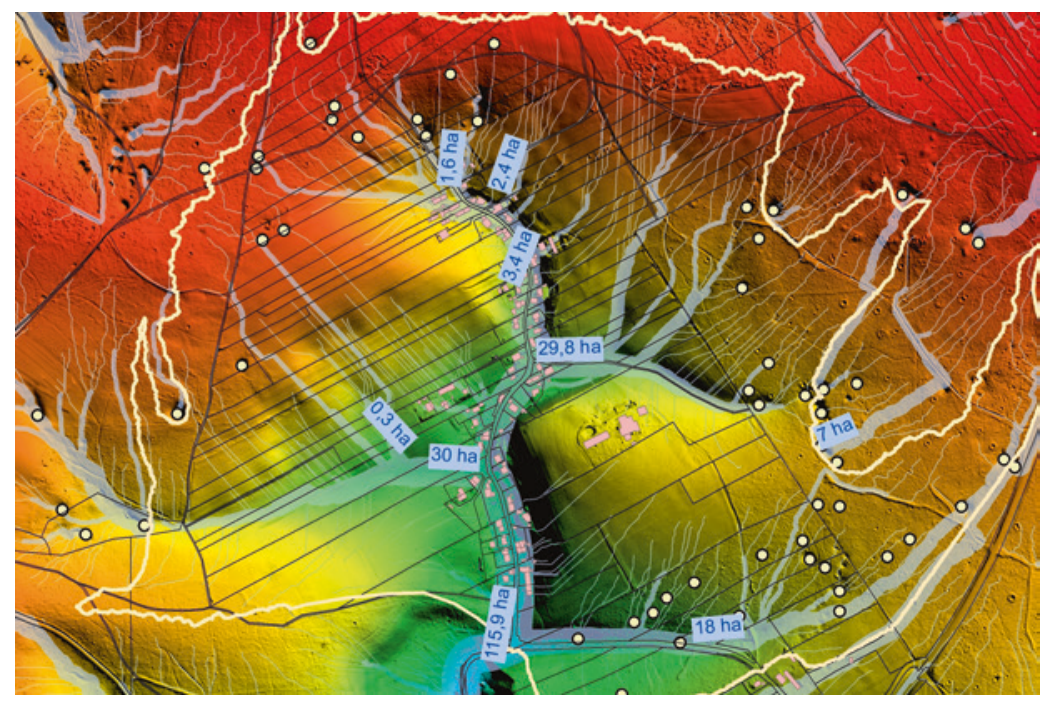

- Abb. 5.6 Ermittlung oberflächlicher Abflusspfade (hellgraue Linien) durch Auswertung hochaufgelöster Geländemodelle (Rasterweite $1 \mathrm{~m}$ ) am Beispiel eines 116 ha großen Einzugsgebietes (weiße Linie) im Malm (weißer Jura) (Höhenunterschied ca. 50 m, mittleres Gefälle 8 \%). Die Breite der hellgrauen Linien wurde relativ zur Größe des angeschlossenen Einzugsgebietes skaliert. Die weißen Punkte markieren die Lage von Dolinen. Schwarze Linien markieren die Flurstücksgrenzen, die Färbung die Höhe über NN. (Quelle: H\&S Ingenieure Freising) 
Aussagen und hydraulische Berechnungen erforderlich, z. B. zur Ermittlung von Scheitelwerten oder Abflussvolumina, müssen der Verlauf der Fließpfade und ihre (hydraulischen) Kenngrößen (Länge, Querschnittsgeometrie, Rauheit und Gefälle) erfasst werden.

\subsubsection{Hydraulische Grundlagen}

Wesentlich ist die Abflussrate (q). Sie ergibt sich durch Multiplikation der mittleren Fließgeschwindigkeit (v) mit der durchflossenen Querschnittsfläche (A):

$$
q=v \cdot A
$$

Wobei

q - Abflussrate $\left(\mathrm{m}^{3} \mathrm{~s}^{-1}\right)$

$v$ - Mittlere Fließgeschwindigkeit $\left(\mathrm{m} \mathrm{s}^{-1}\right)$

A - Durchflossene Querschnittsfläche $\left(\mathrm{m}^{2}\right)$

Wird die Abflussrate durch die Fläche des dazugehörigen Einzugsgebietes $A_{E Z G}\left(m^{2}\right)$ geteilt, resultiert nach Kürzen der Einheiten die mittlere Abflussspende (z. B. $\mathrm{mm} \mathrm{h}^{-1}$ ) des Einzugsgebietes.

$$
q_{s}=3,6 \cdot q / A_{E Z G}
$$

Wobei

q - Abflussrate $\left(\mathrm{m}^{3} \mathrm{~s}^{-1}\right)$

$A_{E Z G}$ - Einzugsgebietsgröße $\left(\mathrm{km}^{2}\right)$

Abflussspenden lassen sich gut mit der Niederschlagsintensität vergleichen, da beide Größen über die gleiche Einheit verfügen. Die Verwendung von Abflussraten oder -spenden ist daher günstiger als die Verwendung von Wasserständen, die immer abhängig von der durchflossenen Querschnittsfläche und somit ortsabhängig sind.

Aus Gl. 5.1 folgt, dass sich die Fließgeschwindigkeit erhöht, wenn die durchflossene Querschnittsfläche abnimmt, und umgekehrt. Sind die Fließwege bekannt, lässt sich diese Gleichung auf alle Abschnitte des

Fließpfades mit relativ einheitlicher Geometrie, Gefälle und Rauheit von der Wasserscheide bis zum Bezugspunkt anwenden (vgl. - Abb. 3.6). Dabei müssen nicht alle (ähnlichen) Fließeinheiten innerhalb eines Abschnitts erfasst werden, sondern es genügt eine repräsentative Einheit. Im Folgenden wird unter Fließpfad der gesamte Fließweg von der Wasserscheide bis zum Bezugspunkt an einer repräsentativen Stelle verstanden; unter Abschnitt (oder Zone) wird ein Teil des Fließpfades mit charakteristischem Fließverhalten verstanden, z. B. der Abschnitt mit schichtförmigem Abfluss oder der Abschnitt mit Rillenabfluss (vgl. - Abb. 3.6); unter repräsentativer Einheit wird z. B. eine typische Rille innerhalb des Abschnitts mit rillenförmigem Abfluss verstanden oder ein Grabenabschnitt mit relativ einheitlichem Gefälle, Querschnitt und Oberfläche.

Durch Division der Fließstrecke (l) mit der mittleren Strömungsgeschwindigkeit ergibt sich die durchschnittliche Fließzeit.

$$
t=\frac{l}{60 v}
$$

mit

t - Fließzeit (min)

I - Fließstrecke (m)

60 - Umrechnungskonstante von $\mathrm{s}$ in $\mathrm{min}$

Aus der Gleichung folgt, dass jede Verlängerung der Fließstrecke die Hochwasserwelle mindert und zwar in zweifacher Art und Weise. Einerseits, weil der Weg länger wird, und andererseits, weil sich bei längerem Weg (und gleichbleibender Höhendifferenz) das Gefälle und damit die Abflussgeschwindigkeit reduziert.

Die Verlängerung der Fließstrecke ist daher das erste wesentliche Prinzip einer hochwassermindernden Fließweggestaltung. Zwei weitere werden folgen. 


\subsubsection{Die Geschwindigkeits- methode zur Bestimmung der Konzentrationszeit}

Die hier dargestellten hydraulischen Grundlagen bilden das Fundament zur Bestimmung der Konzentrationszeit $\quad\left(t_{C}=\right.$ time to concentration). Wesentliche Einflussgrößen sind Gefälle, Ausmaß der Abflussbündelung, sowie Rauheit und Art des Fließpfades. Zusammen mit einer Schätzung des Abflussvolumens erlaubt die Konzentrationszeit, Scheitelabflüsse und Abflussganglinien abzuschätzen bzw. zu konstruieren. Ihr kommt dadurch für viele Bemessungsaufgaben eine hohe Bedeutung zu.

Für Planungszwecke hinreichend wird die Konzentrationszeit für einen definierten Punkt vereinfachend als Konstante angenommen, obwohl sie von Regen zu Regen variiert [18, 19]. Ist die Spannweite der Konzentrationszeit von Bedeutung, kann sie ermittelt werden, indem verschiedene Verfahren verwendet oder die Parameter einzelner Methoden innerhalb sinnvoller Wertebereiche verändert werden. Zur Bestimmung der Konzentrationszeit existieren zahlreiche hydraulische und empirische Methoden [20]. Am wichtigsten für kleine Einzugsgebiete ist die Geschwindigkeitsmethode [21]. Sie basiert auf physikalischen Gesetzmäßigkeiten und berücksichtigt, dass der Fließpfad aus unterschiedlichen Abschnitten besteht. Die hydraulischen Eigenschaften entlang des kompletten Fließpfades werden abgebildet. Dadurch ist sie empirischen Ansätzen überlegen und Mittel der Wahl für kleine Einzugsgebiete und für Planungsaufgaben, bei denen es nicht darauf ankommt, jeden einzelnen Regen bestmöglich zu schätzen, sondern das Zusammenspiel verschiedener Landschaftselemente möglichst optimal $\mathrm{zu}$ gestalten. Weniger oder nicht geeignet ist die Geschwindigkeitsmethode, wenn Prozesse wie Wellenüberlagerungen, Rückstau, Ausuferungen oder die Ausbreitung von Sättigungsflächen stark die Fließzeit beeinflussen. Diese Prozesse werden nicht erfasst. Für größere Einzugsgebiete und hydraulisch komplexe Fließstrecken mit flachen, ausgedehnten Überschwemmungsbereichen ist der Ansatz daher weniger geeignet.

Die Geschwindigkeitsmethode setzt voraus, dass zunächst der Bezugspunkt bestimmt und ausgehend davon der (hydraulisch) längste, hinsichtlich der Nutzung typische Fließpfad bis zur Wasserscheide bestimmt wird (als Beispiel siehe - Abb.6.2). Ist der längste Fließpfad bekannt, muss er in näherungsweise einheitliche Abschnitte untergliedert werden. In kleinen Einzugsgebieten oberhalb der Gewässer III. Ordnung sind das typischerweise Schichtabfluss, Abfluss in Rinnen und Rillen, Hangmuldenabfluss und das Fließen in Gräben und kleinen Bächen (• Abb. 3.6). Ist diese Untergliederung erfolgt, wird für jeden Abschnitt die mittlere Fließgeschwindigkeit und aus der Länge des Abschnitts die jeweilige Fließzeit abgeschätzt. Die hydraulischen Eigenschaften und die Bestimmung der Fließgeschwindigkeit in unterschiedlichen Abschnitten des Fließpfades sind im Folgekapitel und im - Anhang 8.2 beschrieben. Die Konzentrationszeit ergibt sich dann aus der Summe der Fließzeiten der einzelnen Abschnitte $(\triangleright$ Gl. 5.4):

$$
t_{C}=t_{t 1}+t_{t 2}+\ldots+t_{t n}
$$

mit

$t_{c}$ - Konzentrationszeit ( $h$ )

$t_{t n}$ - Fließzeit im Abschnitt $n(h)$

\subsubsection{Fließgeschwindigkeits- bestimmung mithilfe der Gauckler-Manning-Strickler- Gleichung}

Die mittlere Fließgeschwindigkeit ergibt sich nach > Gl. 5.1 aus dem Quotienten von Abflussrate und durchflossener Querschnittsfläche. Selbst in dauerhaft wasserführenden Gerinnen ist die Bestimmung der beiden Parameter aufwendig. Für Planungsaufgaben und insbesondere für Gerinneabschnitte, die nicht dauerhaft Wasser führen, ist es daher einfacher, die Fließgeschwindigkeit mit der empirischen Fließgleichung zu berechnen, die Gauckler, 
Manning und Strickler unabhängig voneinander Ende des 19. und Anfang des 20 Jahrhundert abgeleitet haben (GMS-Gleichung). Die GMS-Gleichung berücksichtigt Neigung, Fließwiderstände und Geometrie des Abflusspfades - und damit alle hydraulischen Faktoren, die den Abflussprozess wesentlich beeinflussen.

$$
v=R^{\frac{2}{3}} \cdot J^{\frac{1}{2}} \cdot k
$$

mit

R - Hydraulischer Radius ( $m$ )

J - Gefälle (-)

k - Rauheitsbeiwert $\left(\mathrm{m}^{1 / 3} \mathrm{~s}^{-1}\right)$

Die GMS-Gleichung ist für viele praktische Fragen hinreichend genau und deshalb heute ein international gültiges Standardverfahren. Sie wird hier für alle Abflusstypen verwendet (einschließlich Schichtabfluss und flachen konzentrierten Abfluss), obwohl die Gleichung im engeren Sinne nur für gleichförmigen turbulenten Abfluss in offenen Gerinnen gilt. Die GMS-Gleichung ist gleichermaßen geeignet, die Fließzeit einzelner Gewässerteilstrecken, die Konzentrationszeit eines Gebietes oder auch den Einfluss einer Maßnahme zum Bremsen des Abflusses entlang des Fließpfades abzuschätzen.

Der hydraulische Radius berücksichtigt die Strömungswiderstände, die sich aus der Geometrie des Fließpfades ergeben. Er ergibt sich aus dem Verhältnis von durchflossener Querschnittsfläche A und benetztem Umfang U. Letzterer entspricht dem Umfang des Gerinnebzw. Fließpfadquerschnittes, der in Kontakt zum Abfluss steht. Je größer der benetzte Umfang im Verhältnis zur Querschnittsfläche, desto kleiner R bzw. desto höher die, aus der Fließpfadgeometrie resultierende, Reibung und damit desto langsamer der Abfluss.

$$
R=\frac{A}{U}
$$

mit

$\mathrm{U}$ - Benetzter Umfang (m)

A - Durchflossene Querschnittsfläche $\left(\mathrm{m}^{2}\right)$

\begin{tabular}{ccc}
\hline Fließabschnitt & $\begin{array}{c}\text { Typische } \\
\text { Form }\end{array}$ & $\begin{array}{c}\text { Typischer } \\
\text { hydraulischer } \\
\text { Radus (cm) }\end{array}$
\end{tabular}

Schichtabfluss

0,2

Rillen- und

Rinnenabfluss

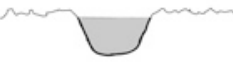

4

Hangmulden-

abfluss

Rohre

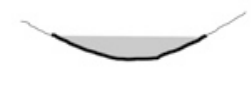

10

25

Gräben
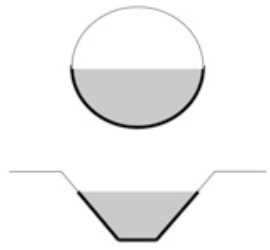

25

Bäche und kleine Gewässer

350

- Abb. 5.7 Typische Formen des Abflusses in aufeinanderfolgenden Fließabschnitten und zugehörige hydraulische Radien (fette Linie = benetzter Umfang, schattierte Fläche $=$ durchflossene Querschnittsfläche)

Typische Beispiele für den hydraulischen Radius in den verschiedenen Abschnitten des Fließweges zeigt - Abb.5.7. Um den hydraulischen Radius für unterschiedliche Querschnittsflächen $\mathrm{zu}$ bestimmen, können die in Anhang 8.2.4 zusammengestellten Formeln verwendet werden. Entlang des Fließweges nehmen $\mathrm{R}$ und damit auch die Fließgeschwindigkeit um mehrere Zehnerpotenzen zu. Umso mehr, je stärker der Abfluss eingeengt wird.

Daher erhöhen alle Maßnahmen die Fließgeschwindigkeit, die den Abfluss konzentrieren. Alle Ansätze, die zu einem breitflächigen Abfluss führen, wirken dagegen bremsend. Dies ist das zweite wesentliche Prinzip einer hochwassermindernden Fließweggestaltung. 
Das Gefälle des Fließpfades (J) (eigentlich müsste das Energiegefälle, also das Gefälle des Wasserspiegels verwendet werden) ist ein $\mathrm{Maß}$ für die beschleunigende Kraft. Je steiler das Gefälle, desto schneller der Abfluss und umgekehrt. Das Gefälle kann im Gelände leicht erfasst oder aus topographischen Karten oder digitalen Geländemodellen abgeleitet werden, indem die Höhendifferenz des Fließpfades durch die dazugehörige Länge des Fließpfades dividiert wird. Entsprechend wird das Gefälle in der GMS-Gleichung dimensionslos bzw. in $(\mathrm{m} / \mathrm{m})$ angegeben. Das Gefälle kann in der Praxis (nur) indirekt über die Länge des Fließweges beeinflusst werden, da die Höhendifferenz zwischen Wasserscheide und Bezugspunkt üblicherweise nicht verändert werden kann.

Der Rauheitsbeiwert (k) berücksichtigt den Einfluss der Oberflächenbeschaffenheit des Fließweges. Je rauer der Fließweg, z. B. durch Bewuchs, desto kleiner der k-Wert und desto geringer die Fließgeschwindigkeit. Umgekehrt steigt $\mathrm{k}$, je glatter und gleichmäßiger Sohle und Wandungen des Fließpfades sind. Die höchsten k-Werte haben glattverputzte Betonkanäle, die niedrigsten treten bei heterogen strukturierten oder stark verkrauteten Fließpfaden auf, z. B. verzweigte Fließpfade in Auwäldern oder alpine Wildbäche mit grobem Geröll und Geschiebetrieb. Wichtig ist, dass der Rauheitsbeiwert linear in der GMS-Gleichung eingeht. Dadurch ist die Strömungsgeschwindigkeit direkt proportional $\mathrm{zu} \mathrm{k}$ und entsprechende Sorgfalt bei der Wahl von $\mathrm{k}$ geboten. Auch hat die Rauheit dadurch nach dem hydraulischen Radius den größten Einfluss auf die Abflussgeschwindigkeit.

Das dritte (und vielleicht wichtigste)

Grundprinzip einer hochwasser-

mindernden Fließweggestaltung besteht daher darin, den Fließweg so hydraulisch rau wie möglich zu gestalten.
Die mittlere Fließzeit (in min) entlang eines Abflusspfades ergibt sich, wenn die GMS-Gleichung in Gl. (5.3) eingesetzt wird.

$$
t=\frac{l}{60 R^{\frac{2}{3}} \cdot J^{\frac{1}{2}} \cdot k}
$$

Rauheitsbeiwerte in einem Gewässer sind bestimmbar, wenn Abflussrate oder Fließgeschwindigkeit und Querschnittsfläche bekannt sind. Im Normalfall muss auf Literaturwerte zurückgegriffen werden, die in - Tab. 5.1 für unterschiedliche Beschaffenheit von Sohle und Böschung zusammengetragen sind (Achtung: für Schichtabfluss gelten andere Rauheitsbeiwerte, die in - Tab. 8.2 zu finden sind; die Unterschiede ergeben sich daher, weil beim Schichtabfluss nicht alle Rauheitselemente überflossen werden und weil die durch auftreffende Regentropfen erzeugten Turbulenzen ebenfalls wesentlich zur Rauheit beitragen). Demnach können je nach Oberflächenbeschaffenheit von Gerinnen die k-Werte zwischen 15 und knapp 80 variieren, wenn man die Gebirgswildbäche außen vor lässt. Dies bedeutet, dass man allein mit der Oberflächenbeschaffenheit den Abfluss schon um den Faktor vier beschleunigen oder abbremsen kann, was den Scheitel einer Abflusswasserwelle um den gleichen Faktor erhöht bzw. senkt. • Tab. 5.1 gibt Spannweiten an. Eher niedrige Werte sind dann anzusetzen, wenn die Rauheit untypisch hoch ist (z. B. starke Verbuschung), eher hohe Werte, wenn das Gerinne untypisch glatt ist (z. B. nach einer Grabenräumung mit einem schälenden Werkzeug). Gleichzeitig erlaubt dies auch eine Abschätzung der Unsicherheit. Da die Rauheitswerte linear die Fließgeschwindigkeit beeinflussen, bedeutet eine Spanne von 40...50, dass die Fließgeschwindigkeit gegenüber einem mittleren Rauheitsbeiwert von 45 um $10 \%$ höher oder niedriger ausfallen könnte. Für gegliederte Gewässerbetten oder zur Berücksichtigung von Unregelmäßigkeiten, Änderungen des Querschnitts, Hindernissen 
- Tab. 5.1 Rauheitsbeiwerte für konzentrierten Abfluss in linearen Strukturen oder flächenhaftem Abfluss mit Fließtiefen $>5 \mathrm{~cm}$ bei unterschiedlicher Beschaffenheit der Oberfläche. Zur Darstellung typischer Spannweiten ist die Rauheit in Bereichen „von" ... „bis" angegeben. Analog wird die Oberflächenbeschaffenheit (Spalte 1) vielfach für unterschiedliche Zustände angegeben. Die niedrigere "von"Rauheitswert bezieht sich dann auf den rauen, der höhere "bis"-Rauheitswert auf den glatteren Zustand. Rauheitsbeiwerte für Schicht- und Rillenabfluss (Fließtiefen $\leq 3 \mathrm{~cm}$ ) können • Tab. 8.2 im Anhang entnommen werden

\begin{tabular}{|c|c|c|}
\hline Oberflächenbeschaffenheit & k-Wert $\left(\mathrm{m}^{1 / 3} \mathrm{~s}^{-1}\right)$ & Quelle \\
\hline \multicolumn{3}{|l|}{ Natürliche und naturnahe Gerinne } \\
\hline $\begin{array}{l}\text { Wildbach mit sehr grobem Fels und Geröll, } \\
\text { Geschiebe in Bewegung, rascher Wechsel von } \\
\text { Becken, Abstürzen und Schussrinnen }\end{array}$ & $9 \ldots 22$ & {$[24-28]$} \\
\hline $\begin{array}{l}\text { Wildbach mit grobem Geröll und Steinen, } \\
\text { Geschiebe überwiegend ruhendend }\end{array}$ & $25 \ldots 28$ & {$[25,27-29]$} \\
\hline $\begin{array}{l}\text { Gewässer mit unregelmäßiger Sohle und } \\
\text { Böschung, je nach Verkrautung, Wurzeln und } \\
\text { Geschiebetrieb }\end{array}$ & $25 \ldots 35$ & {$[25,27-30]$} \\
\hline $\begin{array}{l}\text { Bäche und Gräben mit gleichförmiger } \\
\text { Böschung und Gewässersohle (mit Bewuchs } \\
\ldots \text { ohne oder kurzer Bewuchs) }\end{array}$ & $30 \ldots 35$ & {$[30]$} \\
\hline $\begin{array}{l}\text { Gewässersohle aus Schotter, Grobkies oder } \\
\text { Natursteinen }\end{array}$ & $30 \ldots 45$ & [30] \\
\hline $\begin{array}{l}\text { Flussbett mit Gewässersohle aus Mittel- und } \\
\text { Feinkies, wenig Unregelmäßigkeiten }\end{array}$ & $35 \ldots 55$ & {$[25,27,28,30,31]$} \\
\hline $\begin{array}{l}\text { Einheitliches Gewässerbett aus Sand oder } \\
\text { Feinkies mit wenig Unregelmäßigkeiten }\end{array}$ & $50 \ldots 55$ & {$[30]$} \\
\hline \multicolumn{3}{|l|}{ Kanäle, Gräben und künstliche Gerinne } \\
\hline Raue Sohlrampe/-gleiten & $10 \ldots 20$ & [32] \\
\hline $\begin{array}{l}\text { Böschungsbefestigung aus grober Stein- } \\
\text { schüttung und toleriertem Bewuchs }\end{array}$ & $20 \ldots 27$ & {$[30]$} \\
\hline Erdkanal mit sehr starker Verkrautung & $18 \ldots 24$ & {$[26]$} \\
\hline Grobe Steinschüttung ohne Bewuchs & $20 \ldots 30$ & {$[30]$} \\
\hline $\begin{array}{l}\text { Gerinneprofile mit Sohlen aus Sand und Kies. } \\
\text { Ufer ggf. mit Gras bewachsen, vereinzelt } \\
\text { Schilf oder Röhricht (typisch für korrigierte } \\
\text { Gerinne mit flachem Gefälle, steiler Böschung } \\
\text { und teilweise toleriertem Bewuchs) }\end{array}$ & $28 \ldots 32$ & {$[23,24]$} \\
\hline $\begin{array}{l}\text { Einheitliches Profil mit Sohle aus feineren } \\
\text { Substraten (Lehm) und mit kurzem Gras } \\
\text { bewachsener, steiler Böschung (z. B. Kanäle } \\
\text { und Graben mit mäßigem Bewuchs ... frisch } \\
\text { geräumt) }\end{array}$ & $26 \ldots 40$ & {$[23,30]$} \\
\hline $\begin{array}{l}\text { Erdkanal mit Sohle aus Sand und Kies, } \\
\text { gepflasterte Böschung }\end{array}$ & $40 \ldots 50$ & {$[25,27-29]$} \\
\hline
\end{tabular}


- Tab. 5.1 (Fortsetzung)

\begin{tabular}{|c|c|c|}
\hline Oberflächenbeschaffenheit & $k$-Wert $\left(m^{1 / 3} s^{-1}\right)$ & Quelle \\
\hline Erdkanal aus festem Material, glatt & $50 \ldots 60$ & {$[25,26,29]$} \\
\hline Gemauerter Kanal (unverfugt ... verputzt) & $60 \ldots 70$ & {$[26,28]$} \\
\hline Werkkanal aus Asphaltbeton, glattgestrichen & $72 \ldots 77$ & {$[28]$} \\
\hline \multicolumn{3}{|l|}{ Rohre } \\
\hline $\begin{array}{l}\text { Betonrohr, zusammengesetzt mit } \\
\text { geschlossenen Fugen }\end{array}$ & $85 \ldots 95$ & bauformeln.de ${ }^{a}$ \\
\hline Asbest-Zementrohr (je nach Alter) & $85 \ldots 100$ & bauformeln.de ${ }^{a}$ \\
\hline Stahlrohr (alt/verrostet ... neu/glatt) & $60 \ldots 100$ & - bauformeln.de ${ }^{\mathrm{a}}$ \\
\hline Tonrohr & $70 \ldots 85$ & Expertenschätzung \\
\hline PVC-Rohr & $90 \ldots 100$ & Expertenschätzung \\
\hline
\end{tabular}

Breitflächiges Fließen auf Flächen und Wegen mit unbefestigter, verdichteter oder versiegelter Oberfläche (Fließtiefe $>5 \mathrm{~cm}$ )

\begin{tabular}{|c|c|c|}
\hline $\begin{array}{l}\text { Asphalt und grober Beton, z. B. befestigte } \\
\text { Fahr- und Wirtschaftswege, Parkplätze }\end{array}$ & $50 \ldots 70$ & Expertenschätzung \\
\hline $\begin{array}{l}\text { Feines, festes ggf. verdichtetes Material, z. B. } \\
\text { Grünwege }\end{array}$ & $40 \ldots 45$ & Expertenschätzung \\
\hline $\begin{array}{l}\text { Wassergebundene Decke, z. B. geschotterte } \\
\text { Fahr- und Wirtschaftswege }\end{array}$ & $45 \ldots 50$ & Expertenschätzung \\
\hline Grobkies, Gittersteine, Pflaster & $35 \ldots 50$ & Expertenschätzung \\
\hline
\end{tabular}

Breitflächiges Fließen in Gewässervorländern, bestehend aus landwirtschaftlichen Flächen, Auen und Wäldern (Fließtiefe $>5 \mathrm{~cm}$ )

\begin{tabular}{|c|c|c|}
\hline Bruchwälder & $3 \ldots 6$ & Expertenschätzung \\
\hline Weich- und Hartholzauen je nach Unterwuchs & $6 \ldots 15$ & bauformeln.de ${ }^{\mathrm{a}}$ \\
\hline Wälder (Dickung ... Hochwald) & $12 \ldots 30$ & Expertenschätzung \\
\hline $\begin{array}{l}\text { Hochstaudenfluren und Sukzessionsflächen } \\
\text { je nach Dichte des Bewuchses und Jahreszeit }\end{array}$ & $9 \ldots 29$ & bauformeln.de ${ }^{a}$ \\
\hline $\begin{array}{l}\text { Begrünte Tiefenlinien (Grassed Waterway) je } \\
\text { nach Vegetationsmanagement: } \\
\text { - Ähnlich wie Sukzessionsflächen (Hoch- } \\
\text { staudenflur) } \\
\text { - Wiesenähnlich, z. B. jährliches Mulchen }\end{array}$ & $\begin{array}{l}9 \ldots 29 \\
12 \ldots 30\end{array}$ & Expertenschätzung \\
\hline $\begin{array}{l}\text { Acker (Bedeckung >30 \% } \ldots \\
\text { Bedeckung }<10 \% \text { ) }\end{array}$ & $16 \ldots 25$ & Expertenschätzung \\
\hline $\begin{array}{l}\text { Wiese (hohe, dichte Bedeckung z. B. Sommer } \\
\text {... frisch gemäht oder geringe Bedeckung im } \\
\text { Winter) }\end{array}$ & $25 \ldots 45$ & {$[30]$} \\
\hline
\end{tabular}

\footnotetext{
a https://www.bauformeln.de/wasserbau/gerinnehydraulik/rauheitsbeiwerte-nach-strickler/
} 
im Gewässer stehen weitere Verfahren zur Verfügung, mit denen $\mathrm{Zu}$ - oder Abschläge für die Rauheitsbeiwerte ermittelt werden können [23]. Anhaltspunkte zur Bestimmung von Rauheitsbeiwerten ergeben sich durch visuellen Vergleich mit beschrieben Gewässerquerschnitten und -eigenschaften $[23,24]$.

\section{Literatur}

1. Fiener P, Auerswald K, Van Oost K (2011) Spatiotemporal patterns in land use and management affecting surface runoff response of agricultural catchments - a review. Earth-Sci Rev 106:92-104. - https://doi.org/10.1016/j.earscirev.2011.01.004

2. Kistler M, Brandhuber R, Maier H (2013) Wirksamkeit von Erosionsschutzmaßnahmen, Ergebnisse einer Feldstudie. Schriftenr Bayer Landesanst Landwirtsch 8: Freising

3. Brandhuber R, Treisch M, Fischer F, et al (2017) Starkregen, Bodenerosion, Sturzfluten. Schriftenr Bayer Landesanst Landwirtsch 2: Freising

4. Lenz A, Karlstetter M, Knogler F (2006) Sanierung des landschaftlichen Stoffhaushalts durch Ländliche Entwicklung. Teil A: Planungs- und Umsetzungshilfen. Ingenieurbüro Lenz, Ringelai. - https://www.boden-staendig.eu/_Resources/ Persistent/0d8440a6cacc094919d10d19c3ea863e 40ae3513/SanierungdLandschaft|Stoffhaushdurc hLE-AllgTeil.pdf

5. Hösl R, Strauss $P$ (2011) Einfluss von linearen Abflusswegen auf die Effektivität von Gewässerrandstreifen. Mitt Österr Bodenkundlichen Ges 78:23-28

6. Moussa R, Voltz M, Andrieux P (2002) Effects of the spatial organization of agricultural management on the hydrological behaviour of a farmed catchment during flood events. Hydrol Process 16:393-412. > https://doi.org/10.1002/hyp.333

7. Bronstert A, Vollmer S, Ihringer J (1995) Die Bedeutung von Flurbereinigungsmaßnahmen für das Abflussverhalten von Starkniederschlägen in ländlichen Gebieten. Wasser Boden 47:29-46

8. Machl T, Ewald W, Donaubauer A, Kolbe TH (2016) Entwicklung eines Werkzeugs zur landesweit flächendeckenden Analyse landwirtschaftlicher Transportbeziehungen in Bayern. Z Geodäsie Geoinf Landmanagement 3:197-205

9. Bucher B, Demuth S (1986) Vergleichende Wasserbilanz eines flurbereinigten und eines nicht flurbereinigten Einzugsgebietes im Ostkaiserstuhl für den Zeitraum 1977-1980. Deutsche Gewässerkdl Mitt 29:1-4, Koblenz
10. Luft G, Morgenschweis G (1984) Zur Problematik großterrassierter Flurbereinigung im Weinbaugebiet des Kaiserstuhls. Z Kulturtechnik Flurberein 25:138-148

11. Tessier D (1991) Influence de travaux d'ameliorations foncieres sur le regime hydrologique des petits cours d'eau. Ecole Polytechnique Federale de Lausanne

12. Hölzel H, Diekkrüger B (2012) Predicting the impact of linear landscape elements on surface runoff, soil erosion, and sedimentation in the Wahnbach catchment, Germany. Hydrol Process 26:1642-1654. $>$ https://doi.org/10.1002/hyp.8282

13. Luft G, Vogelbacher A (1985) Modellrechnungen zum Einfluß von Großterrassierungen auf den Abflußprozeß. Z Kulturtechnik Flurberein 26:1-12

14. Hösl R, Strauss P, Glade T (2012) Man-made linear flow paths at catchment scale: identification, factors and consequences for the efficiency of vegetated filter strips. Landsc Urban Plan 104:245-252. > https://doi.org/10.1016/j. landurbplan.2011.10.017

15. Lenz A, Karlstetter M, Knogler F (2006) Sanierung des ländlichen Stoffhaushalts durch Ländliche Entwicklung. Teil B: Fallbeispiel Schwimmbach. Ingenieurbüro Lenz, Ringelai

16. Tempel M (2006) Abflussverhalten kleiner, forstlich genutzter Bacheinzugsgebiete am Beispiel des Einzugsgebietes des Oberen Gräfenbaches im Soonwald / Hunsrück. Dissertation, Johannes Gutenberg-Universität, Mainz

17. Berger C, Schulze M, Rieke-Zapp D, Schlunegger F (2010) Rill development and soil erosion: a laboratory study of slope and rainfall intensity. Earth Surf Process Landforms 35:1456-1467. - https://doi.org/10.1002/esp.1989

18. McCuen RH (2009) Uncertainty analyses of watershed time parameters. J Hydraul Eng 14:490-498. > https://doi.org/10.1061/(ASCE) HE.1943-5584.0000011

19. Grimaldi S, Petroselli A, Tauro F, Porfiri M (2012) Time of concentration: a paradox in modern hydrology. Hydrol Sci J 57:217-228. > https://doi. org/10.1080/02626667.2011.644244

20. Gericke OJ, Smithers JC (2014) Review of methods used to estimate catchment response time for the purpose of peak discharge estimation. Hydrol Sci J 59:1935-1971. > https://doi.org/10.1080/026266 67.2013 .866712

21. Natural Resources Conservation Service (2010) Time of concentration. In: National Engineering Handbook. Part 630 Hydrology, chapter 15. US Department of Agriculture, Washington

22. Landesanstalt für Umweltschutz BadenWürttemberg (2003) Hydraulik naturnaher Fließgewässer. Teil 1. Grundlagen und empirische hydraulische Berechnungsverfahren. Karlsruhe 
23. Hicks DM, Mason PD (1998) Roughness characteristics of New Zealand rivers. National institute of water and atmospheric research. Christchurch, New Zealand

24. Spreafico M, Hodel HP, Kaspar H (2001) Rauheiten in ausgesuchten schweizerischen Fliessgewässern. Serie Wasser. Bundesamt für Wasser und Geologie, Eidgenössisches Departement für Umwelt. Verkehr, Energie und Kommunikation, Bern

25. Rössert R (1994) Hydraulik im Wasserbau. Oldenbourg Wissenschaftsverlag, München

26. Bollrich G, Peißler G (1996) Technische Hydromechanik. Verlag für Bauwesen, Dresden

27. Naudascher E (1992) Hydraulik der Gerinne und Gerinnebauwerke. Springer, Wien
28. Schröder W, Euler G, Schneider F, Knauf D (1994) Grundlagen des Wasserbaus. Werner-Verlag, Düsseldorf

29. Lange G, Lecher K (1993) Gewässerregelung, Gewässerpflege. Paul Parey, Hamburg

30. Knauf D (2003) Zusammenhang zwischen Rauheitsbeiwerten nach Gauckler-Manning-Strickler und den äquivalenten Rauheitsbeiwerten nach Prandtl-Colebrook im hydraulisch rauen Bereich. Wasser Abfall 4-5:28-30

31. Strickler A (1924) Beiträge zur Frage der Geschwindigkeitsformel und der Rauhigkeitszahlen für Ströme, Kanäle und geschlossene Leitungen. Schweiz Bauztg 83:265-269

32. Schröder J (2000) Sohlgleiten im Einzugsgebiet der Leine. Leineverband, Göttingen

Open Access Dieses Kapitel wird unter der Creative Commons Namensnennung 4.0 International Lizenz ( $>$ http://creativecommons.org/licenses/by/4.0/deed.de) veröffentlicht, welche die Nutzung, Vervielfältigung, Bearbeitung, Verbreitung und Wiedergabe in jeglichem Medium und Format erlaubt, sofern Sie den/ die ursprünglichen Autor(en) und die Quelle ordnungsgemäß nennen, einen Link zur Creative Commons Lizenz beifügen und angeben, ob Änderungen vorgenommen wurden.

Die in diesem Kapitel enthaltenen Bilder und sonstiges Drittmaterial unterliegen ebenfalls der genannten Creative Commons Lizenz, sofern sich aus der Abbildungslegende nichts anderes ergibt. Sofern das betreffende Material nicht unter der genannten Creative Commons Lizenz steht und die betreffende Handlung nicht nach gesetzlichen Vorschriften erlaubt ist, ist für die oben aufgeführten Weiterverwendungen des Materials die Einwilligung des jeweiligen Rechteinhabers einzuholen. 